\title{
Mobile Crowd Sensing for Hypertensive Patient
}

This paper was downloaded from TechRxiv (https://www.techrxiv.org).

\section{LICENSE}

CC BY 4.0

SUBMISSION DATE / POSTED DATE

09-04-2020 / 10-04-2020

\section{CITATION}

Patel, Ankit; Fiaidhi, Jinan; Kapadia, Harsh (2020): Mobile Crowd Sensing for Hypertensive Patient. TechRxiv. Preprint. https://doi.org/10.36227/techrxiv.12101409.v1

DOI 


\section{MCS: Mobile Crowd Senesing For Hypertensive Patients}

\author{
Ankit Patel \\ Master's in Computer Science \\ Lakehead University \\ Thunder Bay, Canada \\ apate157@lakeheadu.ca
}

\author{
Harsh Kapadia \\ Master's in Computer Science \\ Lakehead University \\ Thunder Bay, Canada \\ hkapadia@lakeheadu.ca
}

\author{
Prof. Dr. Jinan Fiaidhi \\ Faculty of Computer Science \\ Lakehead University \\ Thunder Bay, Canada \\ jfiaidhi@lakeheadu.ca
}

\begin{abstract}
Nowadays, smartphones and other sensing devices are becoming people's everyday gadgets. The collective intelligence of human and machine along with dominant sensing, computing and communication devices are able to solve real-time problems with ease. Mobile Crowd Sensing is an emerging technology which has evolved with a capability of sensing and networking collective data using mobile devices and sensors via volunteers. The application of MCS in healthcare sector is capable of diagnosing and tracking the severity of disease by using collective data. In this article a system is proposed that collects variety of data from a network of volunteers as well as patients and use them to diagnose the disease called Hypertension. The main aim is to detect Hypertension without considering Blood Pressure or any other medical test. The proposed method mainly focuses on generating huge database which contains symptoms of Hypertensive patients. In MCS environment, another key component is providing incentive to volunteers in-order to motivate them to take part in data collection phase. Feedback is given to the user about their current health status in form of incentive which is generated using Machine Learning technique like Random Forest Classifier.
\end{abstract}

Keywords-Mobile Crowd Sensing, MCS, Machine Learning, Hypertension, Healthcare, Incentive, Random Forest Classifier, Word2Ved

\section{INTRODUCTION}

In recent years, as a result of advancement in the fields of Internet of things, Network, mobile and wireless technologies, the connectivity between people via smartphones and other communication devices has improved up to great extent. The efficiency and capability of network has opened the gateway of transferring large amount of data from one corner of the world to other within fractions of seconds. This has led to an evolution of Crowd Sensing Technology [1].

Mobile Crowd Sensing is an emerging environment which deals with sensing and sourcing huge amount of data based on variety of mobile devices with sensing capabilities. This enables acquiring geographical and atmospheric data like current location, humidity, temperature, wind speed, etc. Also, the MCS technology is capable of sensing technical data, medical data, educational data, etc. and geo-source the meaningful information \& knowledge extracted from the sensed data [2].

Recent advancement in the field of Internet of Things and sensor networks has provided a wide support to research work in fields like healthcare, education, environmental scenario. Monitoring the large-scale phenomenon like predicting weather or any other events cannot easily be carried by a single or a group of humans. MCS paradigm can easily achieve success in such large-scale phenomena using wearable devices and sensors $[3,4]$.

Involvement of humans in Crowd sensing environment is one of the major characteristics of MCS. Traditionally, only sensors (steady as well as mobile) and computing devices were participating in data sensing and sourcing process. But human mobility involvement into sensor technology offers exceptional opportunities for both sensing and sourcing of meaningful data transmission.

In article [5], two types of Mobile Crowd Sensing (MCS) sensing paradigms were presented. One which require human involvement and other without active involvement of humans.

i. Participatory sensing: Humans need to actively participate into sensing and sourcing of data via application. Humans are responsible for quality, accuracy and truthfulness of sensed data.

ii. Opportunistic sensing: The system totally eliminates human involvements in the process. The process is autonomous and the sensors and devices are on its own when it comes to sensing data as well as geosourcing it. (e.g. temperature sensors do not require active human involvement in calculating temperature.)

Participatory sensing is used in the proposed work which requires voluntarily involvement of humans in order to achieve high performance in MCS system.

Apart from types of sensing, two types of data transmission paradigm were mentioned in [5].

i. Infrastructure-based transmission: In this type of transmission, the user access sensory data using internet via cellular networks.

ii. Opportunistic transmission: Sensory data is forwarded using short range radio communication between mobile users.

Infrastructure-based as well as Opportunistic transmission is used in this article depending on the availability of network to transfer data in MCS system to serve hypertensive patients. Both, transmission works well with centralized server or infrastructure for communication and management system.

Mobile Crowd Sensing (MCS) system mainly consists of three phases:
i. Data Sensing
ii. Data Geo-Sourcing 
iii.

\section{Feedback (Incentive Mechanism)}

\section{RELATED WORKS}

A. Secure Mobile Crowdsensing Based on Deep Learning [6]:

The quality of multimedia services and security in transmission of sensing data under Internet of Things application is major concern nowadays. The sector like healthcare and traffic monitoring needs more attention from data security point of view. MCS is capable of addressing security threats such as jamming, spoofing and faked sensing attacks during both sensing and information exchange processes in large-scale dynamic and heterogeneous networks.

In the article [6], research has been done to investigate secure data transmission in MCS network using Deep Learning (DL) algorithms such as stacked autoencoder, deep neural networks, convolutional neural networks, and deep reinforcement learning. The system also takes care of authentication, privacy protection, faked sensing countermeasures, intrusion detection and anti-jamming transmissions in MCS.

Performance between deep learning based approach and traditional security schemes were evaluated and identified the practicality of all the approached in real-time MCS system.

Experimental results suggest that most of the existing deep learning algorithms like DCNN-based authentication [7] or RNN-based malware detection [8] requires heavy level of computing power which includes expensive graphical processing units (GPUs) and take large amount of time to train the model which cannot be applicable to MCS system. Thus, low cost DL components and distributed system need to be deployed over MCS network to ease the process and benefit the MCS application with security based on deep learning algorithms.

In case of transaction fraud or faked sensing motivation, MCS needs to make decision in fractions of time to provide security. CNN and DQN-based faked sensing must be able to cope up with time constraints in MCS environment. Furthermore, backup security protocols need to be implemented in DL-based security schemes to provide reliable and secure MCS services.

B. Mobile Crowd Sensing Services for Tinnitus Assessment, Therapy and Research [9]:

Mobile Crowdsensing has many different applications and one of the major sectors which is benefited from it is healthcare sector. This article talks about the treating Tinnitus using Mobile Crowd Sensing.

Available treatment for Tinnitus is only applicable to particular subgroup of patients. Rest of the patients are still suffering from it and unable to diagnosed or get treated. Currently, for Tinnitus only clinical trials are the standard instrument for realizing evidence-based medicine. But knowledge-based gathering is limited. Individual treatment decisions can be inferred by generating homogenous groups to obtain significant results.

A method is proposed in this article which works on mobile crowdsensing (MCS) approach for collecting large and ecological valid datasets at low costs. In the project named 'TrackYourTinnitus' which is proposed in this system, collects the data from patients and based on that diagnosis is done. Based on diagnosis, patient is provided with the feedback on the patient's treatment and therapy.

The feedback in form of treatment is provided to patients to motivate them to take part in data collection phase. Future works includes addition of more incentives and features to the application to increase user motivation and thus gathering more valuable data on tinnitus disease.

\section{A Mobile Crowd Sensing Application for Hypertensive Patients [10]:}

The work done in article [10] is the main base for the work done in the proposed MCS application. Mainly this article talks about the implementation of mobile crowd sensing technology to treat the patients with Hypertension disease.

The contribution proposes an implementation of MCS network that can able to collects data from the hypertensive patients and create a huge database of hypertension symptoms using Platform as a Service (PaaS). The main aim is to diagnose hypertension without using the main diagnostic feature of hypertension i.e. the blood pressure.

In order to motivate, participant and patients, a feedback is given to the user on their current health condition based on the input they have provided. This is the incentive mechanism in MCS network and to provide this, machine learning algorithm is used at the backend to classify the disease based on the inputs. Two different techniques were investigated out of which Random Forest algorithm performed better than Multi-Layer Perceptron (MLP) and gives the best result.

To take input ECG signals from the patients, a small Mobile ECG sensing extender is used. Reward system is also implemented to keep a check on reliability of the recorded data from volunteers. The features used in this article to detect hypertension are divided into nine groups which includes Age, Heart Rate and technical parameters.

It is concluded in the research work of the article that, the parameters extracted from ECG are sufficient to make a distinction between the hypertensive and normal status of the patients with satisfactory accuracy.

Their future works includes extension of this MCS application for the detection of cardiovascular problems. 


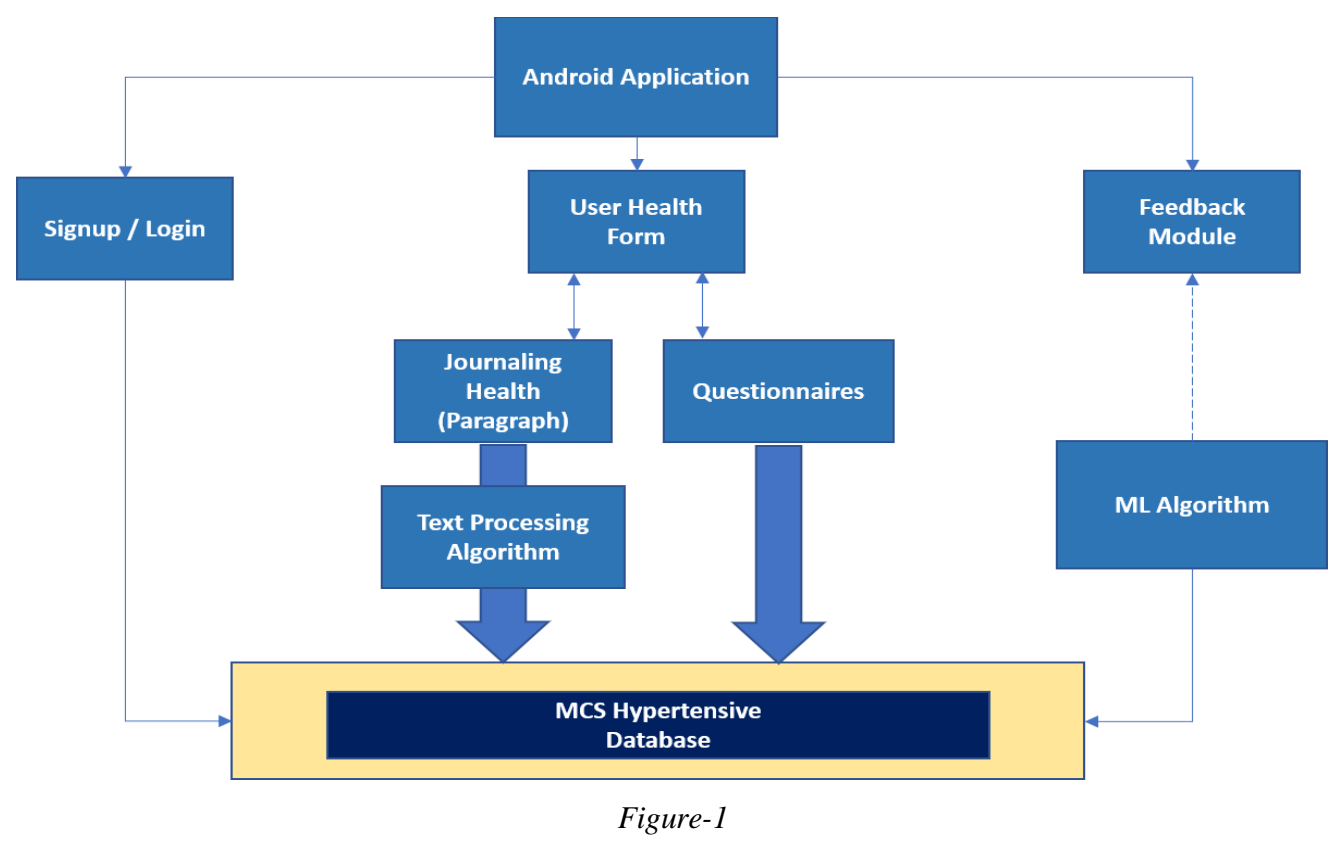

(Application Flow of Proposed MCS System)

An extension to the application can includes GPS coordinates of each subject and automatic acquisition of the corresponding regional meteorology data (temperature, humidity) that affect the cardiovascular parameters. Apart from automatically collected data, the participant would respond to a questionnaire about their subjective feelings (well, headache, dizziness, insomnia); once logged in, the participants could get a feedback about general health attitude of the hypertensive neighbors. This can further motivate to join MCS.

\section{Proposed Methodology}

Integrating Mobile Crowd Sensing technology with Machine Learning techniques on an Android application platform with a backend on Python to treat Hypertensive patients with an ease is the key concept of the proposed MCS application.

\section{A. Project Goal}

The main aim of the proposed MCS application is to collect health specific data from participants and create a huge database of hypertension symptom under Mobile Crowd Sensing environment. Determining the patient's condition regarding Hypertension without considering the Blood Pressure as the most distinctive feature. Provide feedback to MCS volunteers in form of patient's health condition as a part of incentive mechanism.

Application Purpose: To develop and maintain a large database of hypertension symptoms in a variety of circumstances by motivating the patients to feed records under incentive mechanism.

\section{B. Modules in MCS Application}

As mentioned previously, the system is divided into three parts: i. Android Application: Provides the graphical user interface (GUI) to the user through which a participant can login, signup, fill user-health form and get the feedback about their health status. Further in the application, the participant will be provided with two types of health form.

a. Questionnaires: A user will be asked to provide input to questionnaires with a predefined fixed question.

b. Journaling Health: Participant can express their health condition in words i.e. by writing a health journal. To process the words and paragraph, Word2Vec module developed by Google is used.

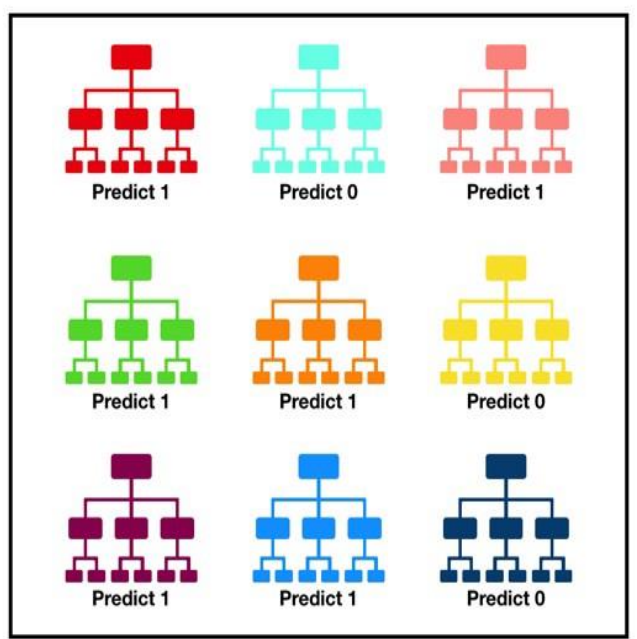

Tally: Six 1s and Three 0s Prediction: 1

Figure-2

(Random Forest Classifier [11]) 
ii. Database: This is a back-end part where database is generated which contains records of health condition of specific participants. Further it can be used for research purpose.

iii. Machine Learning Techniques: Feedback is provided in form of health status of the participant. To generate this feedback, machine learning technique is used which predict the health status of participant based on the given input. Random Forest Classifier is used in the proposed MCS Application.

\section{Algorithms}

i. Random Forest Classifier: Random Forest is an ensemble supervised machine learning (ML) technique [12]. Numbers of decision trees are generated and each of these trees gives different prediction value as shows in Figure-2. Based on bagging and random feature selection, number of decision trees (base classifiers) is generated and majority voting is taken for classification. The classifier in proposed MCS system is used to predict if the participant is suffering from hypertension or not.

Word2Vec: Word2vec offers a unique perspective to the text mining community. It is one of the most popular technique to learn word embeddings using shallow neural network. Word embedding is one of the most popular representation of document vocabulary. Word2Vec converts the words into vetor representation [13]. Using this vector reporesentation, Word2 Vec is capable of fetching the context of a word in a document, semantic and syntactic similarity, relation with other words, etc. A Similarity index will be calculated based on the vector value. If similarity index is greater than threshold value (in our case $30 \%$ ), then particular feature in the dataset will be marked ' 1 ' else ' 0 '.

\section{Application Flow}

The detailed flow of MCS application can be depicted from figure-1. There are two parts in the application flow:

\section{i. Data Collection:}

Participant will be provided with an Android application on their smart phone. To keep track of trustworthiness of the participants, login and signup module is added to the application. It's a basic authentication module added with basic user information. By this way, fake data can be prevented from entering into the database.

After login module, participant will be asked to feed the application with health condition via User Health Form module. To make this part interactive and interesting, user will be provided with two different options to feed health condition as mentioned in the previous section.
In Questionnaires module, user just have to answer list of predefined questions in form of ' 1 ' or ' 0 '. Here ' 1 ' indicates present and ' 0 ' indicates absent. Types of questions asked in this module will be explained later in the next section.

While in Journaling Health module, participant is free to express their health condition in their own words. This is similar to creating the health journal and so is the name of the module.

In case of Questionnaires module, the submitted data will directly be stored into the dataset without any preprocessing or extraction. But in case of Journaling Health module, the paragraph written by user will be provided as an input to the Word2 Vec google algorithm. This algorithm will split the paragraphs and converts each and every word into vector representation. Using this vector representation, a record set will be generated similar to that of Questionnaires module and based on the meaning extracted from Word2Vec algorithm the polarity of the symptoms will be generated i.e. ' 1 ' or ' 0 '. The form generated at the end will be similar to Questionnaires form and the record will be feed to the database.

\section{ii. Incentive Mechanism (Feedback):}

After collecting data from the user, the system will provide feedback as a part of incentive mechanism. The primary goal of Incentive mechanism is to motivate participant to take part in MCS system with a secondary goal of providing diagnostic results of hypertension to the participants.

Machine Learning technique is the key feature of Incentive Mechanism in the MCS system. To diagnose the disease from the input, a prediction is done using Random Forest Classifier based on the participant current record and set of previous records from the database.

When the participant feed the data via User Health Form, that data record will be considered as testing dataset while all the previous records in the database will be considered as training dataset. A model will be trained using Random Forest Classifier on training dataset and will be ready for predicting the output. The testing record will be feed into the trained model and output of the model will be provided as a feedback to the participant. System will provide two types of feedback to participant i.e. 'Tested PositiveHypertension' or 'Tested Negative-Hypertension'.

\section{E. Health Condition Form}

The feature that are used in the Questionnaires module of Health Condition form consists of 17 distinct features and are as follows:
1) Severe Headache
3) Confusion
5) Tension
7) Chest Pain
9) Irregular Heartbeat
11) Insomnia

2) Fatigue

4) Fever

6) Vision Problem

8) Difficult Breathing

10) Blood in the urine

12) Pounding in ENT 
13) Nose Bleeds

15) Blood Spots in Eyes

17) Medicine Side Effects

14) Sweating

16) Anger

\section{CONCLUSION}

The main purpose of the proposed MCS application is to collect health data from the participant using android application. Utilize the collected data to generate large database of Hypertension symptoms and ultimately use that dataset to give feedback to the participants in form of diagnostic results of Hypertension disease. Major challenge is detecting Hypertension disease without the help of main diagnostic feature i.e. Blood pressure or any other medical test. The patient-participants are motivated to participate in MCS system for data sourcing by providing feedback under incentive mechanism. The developed system can be helpful to any hospital for research purpose and can be useful to local people who needs to keep a check on Hypertension disease.

The experimental evaluation of the proposed MCS system shows promising results with good prediction accuracy.

\section{FUTURE WORKS}

- The prototype developed in this article can be used to develop database for any disease not just limited to Hypertension.

- Current Pandemic situation caused by Corona Virus (COVID19) can be tackled using the proposed MCS application by just feeding COVID19 dataset into the system. Instead of waiting for long time for the diagnostic result, ML technique can quickly predict the results based on the dataset.

- Using IoT, it is possible to embed standalone sensors to our android application to provide more medical data and thus getting more accurate diagnostic results.

- Moreover, involving GPS coordinates and atmospheric readings like temperature, humidity, wind speed, pressure, etc. can help extend the performance of MCS system.

\section{ACKNOWLEDGMENT}

Special thanks to Slađana Jovanovi, Milan Jovanovi, Tamara Škori, Stevan Joki, Branislav Milovanovi, Konstantinos Katzis and Dragana Baji for their early work in developing a system for Hypertensive Patients using MCS.

\section{REFERENCES}

[1] Boubiche, D. E., Imran, M., Maqsood, A., \& Shoaib, M. (2018). Mobile Crowd Sensing - Taxonomy, Applications, Challenges, and Solutions. Computers in Human Behavior. doi:10.1016/j.chb.2018.10.028

[2] Ma, H., Zhao, D., \& Yuan, P. (2014). Opportunities in mobile crowd sensing. IEEE Communications Magazine, 52(8), 29-35. doi:10.1109/mcom.2014.6871666

[3] R. K. Ganti, F. Ye, and H. Lei, "Mobile Crowdsensing: Current State and Future Challenges," IEEE Commun. Mag., vol. 49, no. 11, 2011, pp. 32-39.

[4] B. Guo et al., "From Participatory Sensing to Mobile Crowd Sensing," IEEE PerCom Workshops (SCI), 2014.
[5] N. Lane et al., "Urban Sensing Systems: Opportunistic or Participatory?," Proc. HotMobile, 2008, pp. 11-16.

[6] L. Xiao, D. Jiang, D. Xu, W. Su, N. An and D. Wang, "Secure mobile crowdsensing based on deep learning," in China Communications, vol. 15, no. 10, pp. 1-11, Oct. 2018.

[7] C. Shi, J. Liu, H. Liu, et al., "Smart user authentication through actuation of daily activities leveraging WiFi-enabled IoT," in ACM Int'l Symposium on Mobile Ad Hoc Networking and Computing (MobiHoc), Chennai, India, July 2017, pp. 5-15.

[8] T. Shibahara, T. Yagi, M. Akiyama, et al., "Efficient dynamic malware analysis based on network behavior using deep learning," in IEEE Global Commun. Conf. (GLOBECOM), Washington, DC, Feb. 2016, pp. 1-7.

[9] Pryss, R., Reichert, M., Langguth, B., \& Schlee, W. (2015). Mobile Crowd Sensing Services for Tinnitus Assessment, Therapy, and Research. 2015 IEEE International Conference on Mobile Services. doi:10.1109/mobserv.2015.55

[10] Jovanović S, Jovanović M, Škorić T, et al. A Mobile Crowd Sensing Application for Hypertensive Patients. Sensors (Basel). 2019;19(2):400. Published 2019 Jan 19. doi:10.3390/s19020400

[11] https://towardsdatascience.com/understanding-random-forest58381e0602d2

[12] Kulkarni, V. Y., \& Sinha, P. K. (2012). Pruning of Random Forest classifiers: A survey and future directions. 2012 International Conference on Data Science \& Engineering (ICDSE). doi:10.1109/icdse.2012.6282329

[13] Lilleberg, J., Zhu, Y., \& Zhang, Y. (2015). Support vector machines and Word2vec for text classification with semantic features. 2015 IEEE 14th International Conference on Cognitive Informatics \& Cognitive Computing (ICCI*CC). doi:10.1109/icci-cc.2015.7259377 